\title{
Co-channel interference limitations of OFDM communication-radar networks
}

\author{
Martin Braun*, Ralph Tanbourgi and Friedrich K Jondral
}

\begin{abstract}
Orthogonal frequency-division multiplexing (OFDM) communication-radar networks are systems where individual nodes use OFDM signals to both communicate and perform radar simultaneously. As a research subject, such networks are fairly new and lack some research covering fundamental limits. In particular, it is unclear how the reliability of the radar component is affected by a network operation, as several nodes might attempt to access the medium at the same time, thereby increasing interference and reducing the radar capabilities of individual nodes. In this paper, we apply the notion of outage (which was originally introduced for communication networks) to radar networks and introduce the outage probability as a performance metric. Using stochastic geometry, we are able to give tight bounds on the outage probability and demonstrate how this is useful for testing OFDM radar parametrizations and algorithms. It is possible to show that the outage probability is smaller than $1 \%$ for previously suggested OFDM radar parametrizations without having to resort to empirical methods.
\end{abstract}

\section{Introduction}

In 2009, Sturm et al. [1] presented a novel concept for radar systems based on orthogonal frequency-division multiplexing (OFDM) signals, the major innovation being that the radar signal can be used to simultaneously transmit information to other nodes in the range of the radio signal. Unlike previous attempts to combine radar and communication (e.g., [2]), the result of the radar processing is independent from the transmitted data. Also, the radar processing algorithms are not very complex and can easily be implemented on digital signal processing platforms. A possible application of such technology is for automotive systems.

Transceivers which do both radar and communication can thus cooperate and create a communication-radar network $^{\mathrm{a}}$, thereby enhancing the radar as well as the communication features [3].

This also brings disadvantages. Specifically, the problem of interference by simultaneous access to the physical medium by different nodes (i.e., collisions) is exacerbated when compared to networks without radar components: On one hand, such a collision does not only disturb communication but also affects the radar and can thus be

*Correspondence: martin.braun@kit.edu

Communications Engineering Lab, Karlsruhe Institute of Technology, Kaiserstr. 12, Karlsruhe 76137, Germany potentially relevant for traffic safety. On the other hand, automotive radar systems must access the medium on a regular basis to obtain a permanent measurement of the environment, thereby preventing usage of typical carrier sensing schemes. In the domain of pure radar systems for vehicular applications, intra-system interference has been identified as a performance-limiting factor. The European Union (EU) has even launched a project to investigate these matters [4].

From pure communication networks, we know they only work reliably for a limited density of nodes. In radar networks, both communication and radar can fail. In this paper, we aim to provide a new tool for analyzing the performance of OFDM radar networks with respect to the signal parametrization and the network geometry. When the interference level rises, the ability to detect specific objects decreases. We therefore chose to introduce radar network outage as the relevant metric, which describes the situation when a radar network is no longer able to detect a specific object due to interference by others. Choosing successful detection as the main performance metric makes sense for several reasons: It is the first and most basic step in any radar system, and all subsequent operations (range estimation, etc.) depend on it. Also, unlike the detection, the range and Doppler accuracy do not change significantly when operating in a network (cf. [3,5] for resources on OFDM radar accuracy).

\section{是 Springer}

(c) 2013 Braun et al: licensee Springer. This is an Open Access article distributed under the terms of the Creative Commons Attribution License (http://creativecommons.org/licenses/by/2.0), which permits unrestricted use, distribution, and reproduction in any medium, provided the original work is properly cited. 
A common characteristic of vehicular-based networks is high node mobility, which causes the geometrical configuration of nodes to change rapidly - and sometimes also unpredictably. Since an analytical description of these complex spatial fluctuations is difficult, an exact modeling and analysis are not appealing. A remedy to this problem is given by the stochastic geometry framework [6-10], which models the node locations as a realization of a point process, thereby essentially accounting for the spatial dynamics of the network. In this work, we will apply stochastic geometry tools to model the interferer locations. The chosen approach will be instrumental for determining the outage probability of such an OFDM radar network given the physical parameters and providing a definitive answer on how many participants may partake in such a network before the radar system becomes unreliable. Most importantly, we can provide analytical bounds for the probability of a radar outage. At low outage probabilities, which is typically the targeted region of operation in car-safety applications, these bounds coincide with the exact outage probability. This is a significant advantage over current evaluation methods, which can include time-consuming simulations, raytracing setups, or costly measurements.

Using these bounds, we now have an objective metric to analyze various aspects of OFDM radar networks. We give two application examples of our bounds: the quality of the target detection in a multi-target environment and an answer on how the sub-carrier spacing affects the outage probability.

This paper is structured as follows: Section 2 briefly describes the OFDM radar processing used here. The system model for OFDM radar networks and how interference is handled are discussed in Section 3.

In the next two sections, we recapitulate all the basics of OFDM radar networks and clarify which assumptions are made. The new results are derived in Section 4, where we obtain analytical bounds on the outage probability of radar networks, which are further verified by simulations. With these results, we will give a discussion on how this affects the OFDM radar system in Section 5. Section 6 concludes.

\subsection{Previous research}

The topic of mutual interference between radar systems has recently become a focus of research due to the popularization of vehicular radar systems. The previously cited EU research project MOSARIM [4] is an example of how relevant this topic has become.

Radar interference has been researched both analytically and empirically. Brooker [11] provides a very thorough analysis of interference in automotive radar systems at 77 and $94 \mathrm{GHz}$; his metric of choice is the probability of interference. Brooker argues that in case of interference, radar systems cease to work and interference-avoidance techniques must be introduced. This is not a suitable metric for OFDM radar systems (and possibly for any radar system where the interfering radar signals appear as additional white noise).

Goppelt et al. chose the probability of ghost target detection as a figure of merit [12]. However, their results cannot be generalized to OFDM radar, as the derivations are specific to FMCW radar (despite being a commonly applied waveform, OFDM is rarely considered for radar networks). They also lack a random modeling of the interfering signal's attenuation. Similarly, the analysis of Oprisan et al. [13] is also very specific to certain waveforms.

In general, current research focuses on interferenceavoidance and mitigation techniques, which is exemplified by the results from the MOSARIM project, e.g., [14]. Here, OFDM is in fact considered as a method to cope with mutual interference, but further analysis is not given. One suggestion to handle interference instead of avoiding it is given in [15], which is also the only publication which directly researches interference in OFDM radar networks. The paper suggests an interference mitigation technique but only for the very specific scenario of one single interferer.

The difficulty of limiting the scope to a single interferer is also identified by Hischke [16]. He introduces a very useful quantity: The distribution of signal to interference plus noise ratio (SINR) as cause of mutual interference, as a function of the spacing between vehicles, from a given geometry. The results are derived from simulations, though, emphasizing the need for an analytical solution.

The importance of simulations is underlined by the work of Zwick et al. [17], who have done considerable work in the research of mutual radar interference. Their approach is empirical in nature and consists of elaborate software tools packaged under the name Virtual Drive [18]. The results generated are highly useful but emphasize the fact that highly sophisticated simulations are the only means to research radar networks, motivating the derivation of analytical solutions.

In general, there has been little effort to create a 'fundamental radar network theory', analogous to what information theory is for communication networks. Hischke's approach seems the most promising in this respect: If a probability distribution of the SINR could be derived for a given radar interferer density, this would allow a stochastic analysis of the interferer problem. More importantly, it would ground the research on radar networks with theoretical results and provide benchmarks for the empirical results - at this point, there is no theoretical bound for the performance of radar networks. This paper aims to be the first step toward a theoretical understanding of radars operating under mutual interference. 
Stochastic geometry as a tool for research on vehicular networks has previously been suggested in [19], which suggests its suitability in this context.

\section{OFDM radar signal processing principles}

In the following, we present a very brief introduction to OFDM radar ${ }^{\mathrm{b}}$.

\subsection{Nomenclature}

For every radar measurement, one OFDM frame is transmitted. It consists of $M$ consecutive OFDM symbols, with $N$ active sub-carriers. Such a frame is represented by the transmit matrix $\mathbf{F}_{\mathrm{Tx}} \in \mathbb{C}^{N \times M}$, using the notation introduced in [20]. Every column of this matrix represents an OFDM symbol, every row a sub-carrier. The elements $\left(\mathbf{F}_{\mathrm{Tx}}\right)_{k, l}(k=1, \ldots, N, l=1, \ldots, M)$ are symbols from a complex modulation alphabet (e.g., QPSK).

When transmitted, the sub-carriers are separated by a sub-carrier spacing of $\Delta f=1 / T$, with $T$ being the OFDM symbol duration. As $N$ carriers are transporting symbols, the signal bandwidth is $B=N \Delta f$. Converting the matrix into a discrete-time signal is done using the inverse fast Fourier transform on the columns of $\mathbf{F}_{\mathrm{Tx}}$. The transmit signal is extended by a cyclic prefix of duration $T_{G}$. This avoids inter-symbol interference but increases the total OFDM symbol length to $T_{O}=T+T_{G}$. All relevant parameters for the OFDM radar transmitter are listed in Table 1 (these values are discussed in Section 3.5).

Leaving aside synchronization and equalization, receiving OFDM signals is the exact same procedure as the transmission, only in inverse order. The samples corresponding to the cyclic prefix are discarded, and the samples are processed with a fast Fourier transform (FFT). Every FFT output is then assigned to a column of a matrix $\mathbf{F}_{\mathrm{Rx}}$ which represents the received signal.

\subsection{OFDM radar fundamentals}

To extend an OFDM transceiver into a radar system, it must be made sure that during the transmission of every OFDM frame, the receiver is active synchronously, i.e.,

\section{Table 1 Relevant parameters of an OFDM transmitter}

\begin{tabular}{lll}
\hline Parameter symbol & Description & Example value \\
\hline$\Delta f$ & Sub-carrier spacing & $90.9 \mathrm{kHz}$ \\
\hline$T=\frac{1}{\Delta f}$ & OFDM symbol duration & $11 \mu \mathrm{s}$ \\
\hline$T_{G}$ & Cyclic prefix duration & $1.375 \mu \mathrm{s}$ \\
\hline$T_{O}$ & Total OFDM symbol duration & $12.375 \mu \mathrm{s}$ \\
\hline$N$ & Number of active sub-carriers & $1,024($ for $U=1)$ \\
\hline$M$ & OFDM symbols per frame & 256 \\
\hline$f_{C}$ & center frequency & $24 \mathrm{GHz}$ \\
\hline$P_{\mathrm{Tx}}$ & Transmit power & $20 \mathrm{dBm}$ \\
\hline
\end{tabular}

transmitter and receiver use the same local oscillator and, derived from this, an identical clock (this setup describes monostatic radar systems). This ensures that the transmitted signal is delayed at the receiver by the round-trip propagation time of the electromagnetic wave whenever it is scattered back to the original node from a radar target. If the target is moving relative to the radar system, this causes a Doppler shift which appears as a frequency deviation of the received signal relative to the transmitted signal.

Using the matrix notation, it is useful to consider the form of a receive matrix $\mathbf{F}_{\mathrm{Rx}}$ given a transmit matrix. For $H$ reflecting targets ${ }^{\mathrm{c}}$, the receive matrix has the form [20]

$\left(\mathbf{F}_{\mathrm{Rx}}\right)_{k, l}=\sum_{h=0}^{H-1}\left(\mathbf{F}_{\mathrm{Tx}}\right)_{k, l} \cdot b_{h} e^{j 2 \pi l T_{O} f_{D, h}} e^{-j 2 \pi \tau_{h} k \Delta f} e^{j \varphi_{h}}+(\tilde{\mathbf{Z}})_{k, l}$,

which contains the following elements:

- $b_{h}$ is the attenuation of the signal reflected from the $h$-th target; this includes both free space path loss and different reflectivity characteristics (i.e., radar cross sections).

- $\varphi_{h}$ is a random phase shift.

- The Doppler shift causes a frequency offset $f_{D, h}=f_{C} \frac{2 v_{\text {rel, h }}}{c_{0}}\left(v_{\text {rel, h }}\right.$ being the relative speed of the $h$-th target, $c_{0}$ the speed of light). We assume the bandwidth of the signal to be much smaller than the center frequency $B \ll f_{c}$, so we can approximate an identical Doppler shift on all sub-carriers. A frequency-shift of one line of $\mathbf{F}_{\mathrm{Tx}}$ is equivalent to multiplying it with a complex sinusoid $e^{j 2 \pi l T_{O} f_{D, h}}$.

- The round-trip delay $\tau_{h}=\frac{2 r_{h}}{c_{0}}$ (with $r_{h}$ being the range of the $h$-th target) causes a phase rotation of the received symbols depending on the sub-carrier frequency, $e^{-j 2 \pi \tau_{h} k \Delta f}$.

- Additionally, there is white Gaussian noise (WGN), which is represented by the matrix $\tilde{\mathbf{Z}}$.

The matrix $\mathbf{F}_{R x}$ still contains the modulation symbols from the original transmission. These are irrelevant to the radar processing but can be eliminated from Eq. (1) by element-wise division with the (known) transmit matrix, resulting in the radar processing matrix

$$
\begin{aligned}
(\mathbf{F})_{k, l} & =\frac{\left(\mathbf{F}_{\mathrm{Rx}}\right)_{k, l}}{\left(\mathbf{F}_{\mathrm{Tx}}\right)_{k, l}} \\
& =\sum_{h=0}^{H-1} b_{h} e^{j\left(2 \pi\left(l T_{\mathrm{O}} f_{D, h}-k \tau_{h} \Delta f\right)+\varphi_{h}\right)}+\underbrace{\frac{(\tilde{\mathbf{Z}})_{k, l}}{\left(\mathbf{F}_{\mathrm{Tx}}\right)_{k, l}} .}_{=\mathbf{Z}} .
\end{aligned}
$$

For phase-shift keying (PSK) modulation schemes, the noise $\tilde{\mathbf{Z}}$ retains its statistical properties after the division 
[20], as the phase of circular complex Gaussian random variables is uniformly distributed and thus has the same probability distribution after the division. The elements $(\mathbf{Z})_{k, l}$ of $\mathbf{Z}$ are thus still realizations of a WGN process with zero mean and variance $\sigma_{N}^{2}$.

Now, the radar estimation problem can be expressed as a problem of spectral estimation. An OFDM radar algorithm based on Eq. (3) must consist of at least two steps: (1) estimating the number $H$ of sinusoid-pairs and (2) identifying their frequencies, which then translate into distances and relative velocities.

For one-dimensional signals, the optimal way to identify sinusoids in white noise is the periodogram [21]. We therefore extend the periodogram to two dimensions, accounting for the different signatures of the row- and column-wise oscillations, resulting in

$\operatorname{Per}(n, m)=\left|\sum_{k=0}^{N_{\mathrm{Per}}-1}\left(\sum_{l=0}^{M_{\mathrm{Per}}-1}(\mathbf{W})_{k, l}(\mathbf{F})_{k, l} e^{-j 2 \pi \frac{l m}{M_{\mathrm{Per}}}}\right) e^{j 2 \pi \frac{k n}{N_{\mathrm{Per}}}}\right|^{2}$.

The dimensions of this 2D periodogram can be chosen larger than those of $\mathbf{F}, N_{\text {Per }}>N, M_{\text {Per }}>M$, which can be achieved by zero-padding F. This interpolates the periodogram, resulting in a smaller quantization error. The matrix $\mathbf{W}$ is a real tapering window matrix, the choice of which is outside the scope of this work ${ }^{\mathrm{d}}$; unless stated otherwise, we choose a boxcar window $\left((\mathbf{W})_{k, l}=1\right)$.

Figure 1 shows an example of such a periodogram with five targets and matrix dimensions $N_{\mathrm{Per}}=4 \mathrm{~N}, M_{\mathrm{Per}}=4 \mathrm{M}$.
Every pair of sinusoids in $\mathbf{F}$ manifests as a single peak with its center at $\operatorname{Per}(m, n)$. If the periodogram has a peak at indices $(\hat{m}, \hat{n})$, this corresponds to a target at [5]

$$
\hat{r}=\frac{c_{0}}{2 \Delta f} \frac{\hat{n}}{N_{\text {Per }}} \quad \text { and } \quad \hat{v}_{\text {rel }}=\frac{c_{0}}{2 f_{C} T_{O}} \frac{\hat{m}}{M_{\text {Per }}} .
$$

When a maximum distance and relative speed is known, we calculate maximum indices $\hat{m}, \hat{n}$ as

$$
\begin{aligned}
& M_{\max }=\left\lceil\frac{v_{\text {rel,max }}}{c_{0}} \cdot 2 f_{C} T_{O} M_{\text {Per }}\right\rceil, \\
& N_{\max }=\left\lceil\frac{r_{\max }}{c_{0}} \cdot 2 \Delta f N_{\text {Per }}\right\rceil .
\end{aligned}
$$

Because the Doppler (unlike the range) can be both negative and positive, we thus constrain the index ranges of $\operatorname{Per}(n, m)$ to $0 \leq n \leq N_{\max }-1$ and $-M_{\max } \leq m \leq$ $M_{\max }-1$, thereby cropping the periodogram to a smaller size $\left(2 M_{\max }+1\right) \times N_{\max }$.

The choice of $N_{\max }$ and $M_{\max }$ is relevant to the estimation process: Of course, they should not be chosen too small, as valid targets might not be detected, but choosing them too large leads to an increased false alarm rate.

On a separate note, the threshold depends on the noise power, which, as we explain in Section 3.3, is unknown for every received frame and thus must be estimated. The simplest way to do this is to choose a row with index $n_{0}>$ $N_{\max }$ and estimate the variance of this row,

$$
\hat{\sigma}_{N}^{2}=\frac{1}{2 M_{\max }+1} \sum_{m=-M_{\max }}^{M_{\max }-1} \operatorname{Per}\left(n_{0}, m\right)
$$

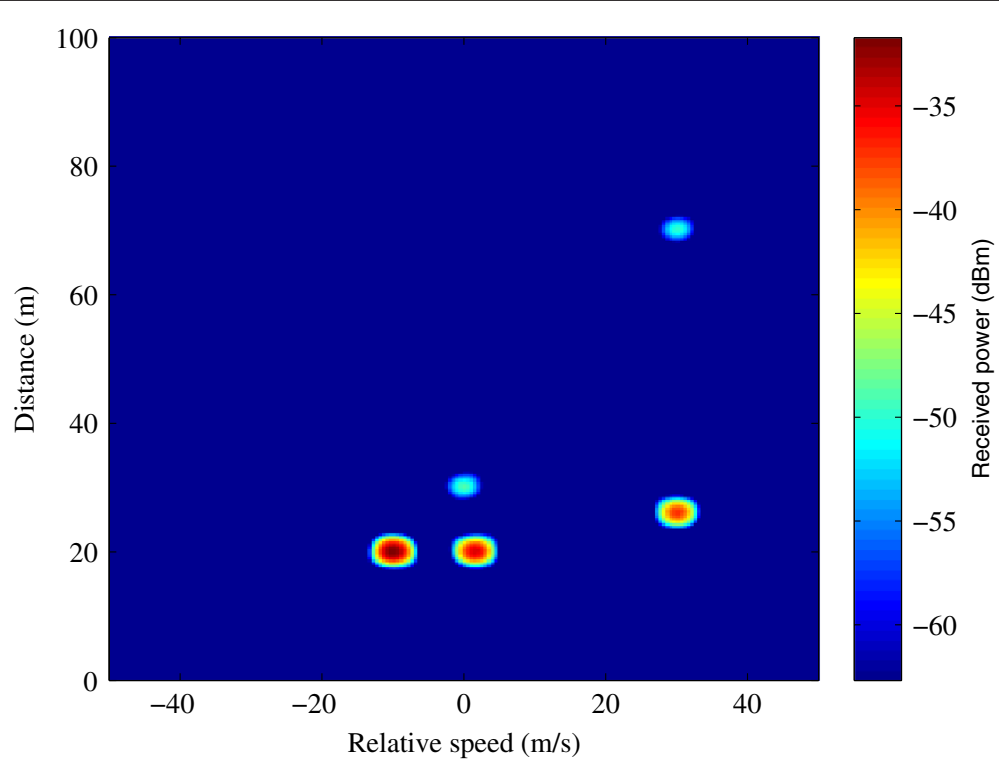

Figure 1 Example of a periodogram $\operatorname{Per}(n, m)$ with $H=5$, all targets having different radar cross section, range, and Doppler. 
which we take as a maximum likelihood estimate for the noise power given a single row of the periodogram.

\subsection{Target detection and false alarm rate}

Having calculated the periodogram, the next step is to identify peaks within the periodogram and to return a list of targets, including their ranges and relative velocities. We call this process target detection.

The majority of detection algorithms rely on a threshold to separate noise from valid signal peaks. This threshold is determined from the noise power as well as from the required false alarm rate. It is commonly desired to keep the false alarm rate at a constant level $p_{F}$. This rate is the probability that any of the $N_{\max } \cdot\left(2 M_{\max }+1\right)$ bins exceed a threshold $\theta$. The noise in the periodogram is exponentially distributed with cumulative density function $F(x)=1-e^{-\frac{x}{\sigma_{N}^{2}}}$, solving for $\theta$ therefore yields a threshold

$$
\theta=\sigma_{N}^{2} \cdot \underbrace{\ln \left(1-\sqrt[N_{\max } \cdot\left(2 M_{\max }+1\right)]{1-p_{F}}\right)}_{c} .
$$

We abbreviate this 'safety factor' with $c$.

For the following derivations, the assumption is made that a target is always detected when its corresponding peak in $\operatorname{Per}(n, m)$ is larger than $\theta^{\mathrm{e}}$. For a given distance $r$ to the target and radar cross section (RCS) $\sigma_{\mathrm{RCS}}$, we can calculate the peak value of the periodogram by applying the pointscatter approximation ([22], Chap. 2), which states that the received reflected power is

$$
P_{\mathrm{Rx}}=\frac{P_{\mathrm{Tx}} G c_{0}^{2} \sigma_{\mathrm{RCS}}}{(4 \pi)^{3} f_{c}^{2} r^{4}} .
$$

Here, $G$ is the combined antenna gain of transmit and receive paths.

As the transmit matrix has unit power, $E\left\{\left|\mathbf{F}_{\mathrm{Tx}}\right|_{k, l}^{2}\right\}=1$, the division (2) does not change the power, $E\left\{|\mathbf{F}|_{k, l}^{2}\right\}=$ $E\left\{\left|\mathbf{F}_{\mathrm{Rx}}\right|_{k, l}^{2}\right\}=P_{\mathrm{Rx}}$. The periodogram finally shifts the entire power into a single bin, leaving the noise power unchanged. Assume the bin index for a target is $n_{0}, m_{0}$, and no noise is present, the peak value of the periodogram becomes

$$
P_{\text {peak }}=\operatorname{Per}\left(n_{0}, m_{0}\right)=P_{\mathrm{Rx}} \cdot N M \text {. }
$$

With noise, $P_{\max }$ is a random variable,

$$
P_{\text {peak }}=\left|\sqrt{P_{\mathrm{Rx}} \cdot N M}+z\right|^{2}
$$

where $z$ is complex, Gaussian distributed with the variance $\sigma_{N}^{2}$.
We simplify the following analysis by approximating $P_{\max }$ with its expected value, given a certain variance:

$$
P_{\text {peak }} \approx \mathbb{E}\left[\max \left\{\operatorname{Per}\left(n_{0}, m_{0}\right)\right\}\right]=P_{\mathrm{Rx}} \cdot N M+\sigma_{N}^{2}
$$

We can justify the approximation by noting that the factor $N M$ is a very large value for typical setups and therefore $P_{\mathrm{Rx}} \cdot N M \gg \sigma_{N}^{2}$ when the interference is low. Only when the noise power becomes too large (e.g., when there is a very high number of interferers, see the following section), this approximation becomes inaccurate. In this region, a radar system becomes highly unusable anyway.

\section{Radar network setup}

The previous section discussed the operation of a single OFDM radar unit. The next step is to describe how these systems work in a multi-user environment. Furthermore, we introduce the concept of outage in OFDM radar networks, which will become the basis of further analyses. When designing an OFDM radar network, we must make sure to satisfy the assumption that all interference can be modeled as AWGN (this becomes relevant for the derivations in Section 4).

\subsection{Time-slotted multi-user access}

When two OFDM signals interfere, we must distinguish two cases: synchronous and asynchronous interference. In the former case, all nodes start transmitting simultaneously, whereas in the latter case, both transmitters may transmit at any given time. This second case is far worse, as the interfering OFDM signal would appear to have a different OFDM symbol duration, and its energy would randomly leak across sub-carriers. This causes additional problems, which are avoided by enforcing simultaneous medium access.

We therefore postulate the following type of multi-user access, which concurs with the system proposed in [23]:

- Access to the medium can only happen at the beginning of a time slot, which is known to all nodes.

- Medium access is allowed on one of $U$ logical channels (as demonstrated in Figure 2). Every channel may only utilise a subset of the OFDM sub-carriers which consist of every $U$-th sub-carrier, starting at sub-carrier $u$, where $u$ denotes the channel number. Note that this changes the OFDM processing only in a way such that the sub-carrier spacing is increased by $U$ and the number of rows in $\mathbf{F}$ is reduced by $1 / U$. Consequently, the power per active sub-carrier is increased by $U$, in order to maintain the same transmit power.

- Every node may randomly access any channel starting at the beginning of any time slot with probability $p_{\mathrm{Tx}}$. While this allows no sophisticated MAC (e.g., 


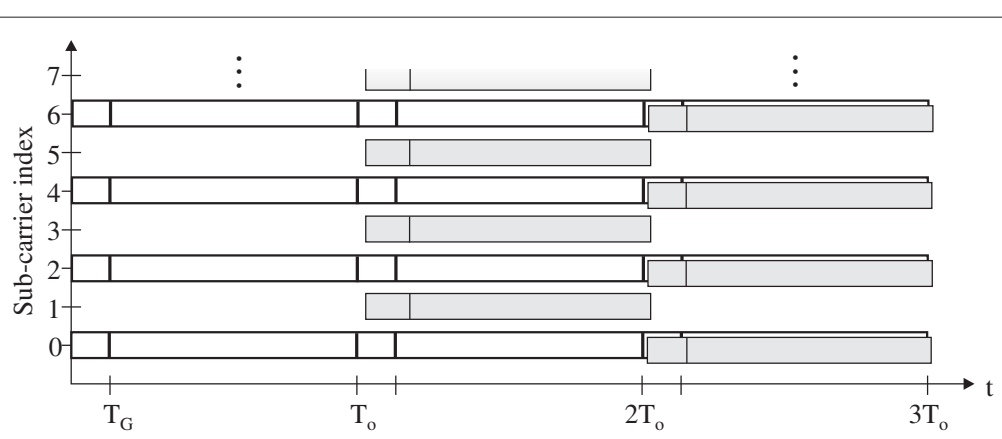

Figure 2 Examples for medium access: There are two channels $(\boldsymbol{U}=\mathbf{2})$. In the first time slot, a node accesses channel 0 , which utilizes the even sub-carriers. In the second slot, two nodes access channels $u=0$ and $u=1$, respectively. Finally, two nodes access the medium on the same channel, causing a collision.

through back-off mechanisms or similar collision avoidance techniques), it allows a radar system to access the medium at regular intervals, which is a requirement for safety-ensuring radar systems.

Having multiple channels in the frequency domain allows for an OFDMA-like multiple access scheme; if two or more nodes access the medium in the same time slot, they only collide if they use the same channel.

From a practical point of view, stipulating synchronous access by time slots requires a global clock. This could be provided by global positioning system (GPS) ${ }^{\text {f }}$. The guard interval $T_{G}$ must then be chosen large enough to allow for clock inaccuracies and different signal arrival times due to different distances to the other radar transmitters.

If clocks differ too much between nodes, minor interference can also be caused by nodes which have chosen a different channel $u$. For this work, we accept a certain inaccuracy of the model and assume perfect orthogonality between channels, as we focus on the definition of radar network outage and how to derive it.

\subsection{Radar network outage}

In communication networks, outage is a common concept to describe the case where an ongoing transmission fails to achieve a given rate. Goldsmith [24] defines outage as the event where SINR (the ratio of received signal power to the sum of noise and interference power) drops below a certain value due to slowly varying, random channel conditions. We can directly transfer this notion to a radar network by defining outage as the case when the reflected power at the receiver drops below a certain threshold due to the interference created by the randomly located nodes.

For a meaningful analysis, we define a reference target as a fixed object at range $r_{\text {Ref }}$ and with a radar cross

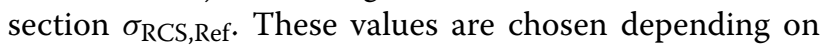
the application at hand (see Section 3.5). Whether an object is detected or not depends on the received power, which is calculated from (10) using the reference target specifications,

$$
P_{\mathrm{Rx}, \mathrm{Ref}}=\frac{P_{\mathrm{Tx}} G c_{0}^{2} \sigma_{\mathrm{RCS}, \mathrm{Ref}}}{(4 \pi)^{3} f_{c}^{2} r_{\mathrm{Ref}}^{4}} .
$$

The outage definition is therefore the same for any object with the same backscattered power $P_{\mathrm{Rx}}$, Ref. The performance of an OFDM radar network is completely defined by the detection of this target.

From Section 2.2, we know that detection is only possible if the peak in the periodogram corresponding to the target has a maximum value larger than the threshold $\theta$, which is a random variable as we consider the interference power to be random. The outage probability is therefore the probability that this peak is smaller than $\theta$,

$$
\begin{gathered}
p_{\text {out }}=\operatorname{Pr}\left[P_{\text {peak }}<\theta\right] \\
\stackrel{(13)}{\approx} 1-p_{D} .
\end{gathered}
$$

By applying the approximation (13), outage probability is complementary to the detection probability $p_{D}$.

This also implies that multi-path propagation of the radar signal does not affect the outage probability and is thus not considered here. A multi-path backscattering might produce additional peaks in the periodogram which do not correspond to true targets, but this is a common problem of all radar systems and must be treated downstream in the processing chain.

\subsection{Interference model}

To model the interference, we use a stochastic model for the interferer geometry. The reference node is located at the origin of a plane. The positions of the other, interfering, nodes follow a stationary two-dimensional Poisson point process (PPP) with density $\lambda^{g}$. Figure 3 illustrates such a scenario. A formal introduction of the specific PPP follows in Section 4.3. 


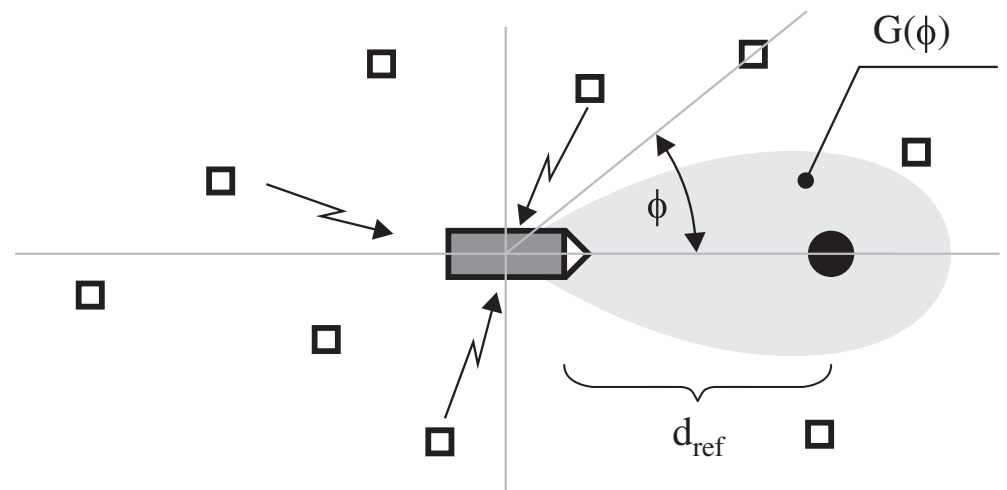

Figure 3 An example of the network topology: the reference node (center) is trying to detect a reference target (circle). Other systems (squares) are randomly distributed and can interfere.

The reason we choose to model the geometry by a PPP is because not only does this provide us with the mathematical tools to analyze such a scenario but also because the main application we have in mind for radar networks is for vehicular technology, where mobility causes a high amount of 'spatial randomness.' Such a spatial model has been shown to properly capture these random spatial dynamics affecting the interference [25].

We emphasize that all nodes represented by this PPP are OFDM transmitters of the same type as the reference node. Because of the homogeneous setup, the results for the reference node are representative for all other nodes as well.

As this is a radar system, we need to be able to determine the azimuth $\phi$ of the targets as well as the range and Doppler. How the radar system implementation solves this problem is irrelevant for this work, what matters is that the angular resolution results in a receiver directivity which can be expressed as azimuth-dependant gain $G(\phi)$. For the transmitters, we assume that they emit omnidirectional so they can communicate with all other nodes.

At this point, we have all the knowledge required to calculate the outage probability for an OFDM radar network with a given network density $\lambda$. To recapitulate, the reason for outage is that whenever the reference node tries to obtain a radar image, a number of interferers might be also transmitting. This increases the interference level and thus the threshold $\theta$.

To show that the network setup still allows the usage of the radar processing from Section 2.2, we must show that the total interference is additive white Gaussian noise (AWGN). Conditioning on a certain spatial configuration, assume we have $I$ interferers, with $\mathbf{F}_{\mathrm{Ix}, i}$ being the transmit frame of the $i$-th interferer. The noise matrix $\mathbf{Z}$ now does not only contain the receiver noise but also energy from the interfering transmit symbols. By assuming synchronous interference (see Section 3.3), we may analyze the total noise matrix element-wise, which then becomes

$$
\left(\mathbf{Z}_{\text {total }}\right)_{k, l}=\mathbf{Z}_{k, l}+\sum_{i=0}^{I-1} \sqrt{b_{i}} \frac{\left(\mathbf{F}_{\mathrm{Ix}, i}\right)_{k, l}}{\left(\mathbf{F}_{\mathrm{Tx}}\right)_{k, l}} e^{j \varphi_{i}} .
$$

Remember that the $\left(\mathbf{F}_{\mathrm{Ix}}\right)_{k, l}$ are zero for interferers which use a different channel than the reference node (assuming perfect orthogonality).

On top of the receiver noise, we now have a sum of complex values with random amplitude and phase; we can model the latter as uniformly distributed within $[0,2 \pi)$. The former is modeled by an exponential path loss,

$$
b_{i}=g_{i} \frac{\beta}{r_{i}^{\alpha}} G(\phi),
$$

where $r_{i}$ is the distance to the origin, $\phi$ the azimuth and $\alpha>2$ the path loss exponent. $\beta$ is a constant attenuation factor, which is assumed to fulfill

$$
\beta=P_{\mathrm{Tx}} \frac{c_{0}^{2}}{(4 \pi)^{2} f_{C}^{2}}
$$

in correspondence with free space path loss. $g_{i}$ is an optional random small-scale power attenuation parameter (caused by fading) with distribution function $F_{\mathrm{g}}(g)^{\mathrm{h}}$; we discuss the cases where $g_{i}=1$ (i.e., no fading), or i.i.d. exponentially distributed with unit mean (Rayleigh fading). This fading parameter covers multipath propagation of the interference signals. A very similar model is also described in [19].

Any fading type can be inserted, as long as its distribution function can be given. Here, Rayleigh fading was chosen as an example for a fading model, as it is a popular choice for the modeling of wireless fading channels and its probability density function (pdf) is mathematically easy to describe. 
In the special case where the modulation has constant amplitude (e.g., as in PSK) and the amplitude is Rayleighdistributed, $\mathbf{Z}_{\text {total }}$ is a sum of normally distributed random variables and therefore is normally distributed by the central limit theorem. For the more general case where the $b_{i}$ follow any distribution (in Eq. (18), we state that they depend on the distance of the interferers to the reference node and are thus not identically distributed), we may still refer to the central limit theorem, which states that a small number of summands (10 to 12) suffice for $\mathbf{Z}_{\text {total }}$ to be approximately Gaussian ([26], Chap. 2).

For the rest of this work, we will omit the index 'total' and use $\mathbf{Z}$ to describe the compound noise with total twosided noise power $\sigma_{N}^{2}+\tilde{Y}$, where $\tilde{Y}$ denotes the random variable representing the total interference power.

\subsection{Clutter}

We have deliberately omitted a modeling of clutter, for two reasons: First, there are many different clutter models, and incorporating one specific model would make our results less versatile. Second, the outage probability is a theoretical boundary depending on the specifications of the reference target, and as such is unaffected by clutter.

This approach is not unusual in vehicular radar applications, where clutter is commonly not treated differently than other targets at the detector; an example would be the detection of a road sign when intended targets are other vehicles. Both these objects register as a backscattering object at the receiver (and therefore as a peak in the periodogram).

\subsection{Parametrization}

In the case where we need to specify the parameters (e.g., for simulations), we use the values given in Table 1, which coincide with the parameters chosen in previous publications [1,27]. These parameters are also discussed in [3], and research on the radar accuracy for a single node (outside of a network) has been done through simulations in [5] and by measurements in [27]. They provide a range resolution of approximately $3.2 \mathrm{~m}$, and a Doppler resolution of approximately $2.5 \mathrm{~m}$, although some modifications of the parametrization (e.g., other window matrices $\mathbf{W}$ ) can degrade this value. For a detailed discussion on the choice of these parameters, we refer to [28].

All other parameters relevant to the network setup are chosen as shown in Table 2.

The target parameters are chosen to work within a vehicular scenario. For the receiver, the noise figure is one compound value encompassing all non-idealities and distortions caused by the amplifiers and the digital signal acquisition. The receiver noise power is then calculated using the given bandwidth $(B=N \Delta f=93 \mathrm{MHz})$ and at a noise temperature of $290 \mathrm{~K}$.
Table 2 Radar network setup

\begin{tabular}{ccc}
\hline & Target parameters \\
\hline$r_{\text {Ref }}$ & & $50 \mathrm{~m}$ \\
\hline$v_{\text {rel,Ref }}$ & $0 \mathrm{~m} / \mathrm{s}$ \\
\hline$\sigma_{\text {RCS,Ref }}$ & $10 \mathrm{~m}^{2}$ \\
\hline$P_{\text {Rx,Ref }}$ & $10 \mathrm{~m}^{2}$ \\
\hline$d_{\max }$ & $300 \mathrm{~m}$ \\
\hline$V_{\text {rel,max }}$ & $150 \mathrm{~m} / \mathrm{s}$ \\
\hline$N_{\max }$ & 187 \\
\hline$M_{\max }$ & 153 \\
\hline & Receiver parameters \\
\hline Noise figure & $10 \mathrm{~dB}$ \\
\hline Thermal noise power & & $-84.2 \mathrm{dBm}$ \\
\hline & Network parameters & 0.01 \\
\hline$p_{F}$ & & 0.1 \\
\hline$p_{\text {Tx }}$ & & \\
\hline
\end{tabular}

As for the network parameters, if we specify a time slot duration of $5 \mathrm{~ms}$, the average medium access rate is $20 \mathrm{~Hz}$, a typical value for automotive radar systems. The false alarm rate is the targeted false alarm rate at the detector; in practical systems, tracking devices or similar systems will further decrease the false alarm rate for practical purposes.

For the antenna gain, we define the width of the antenna beam as $\phi_{0}$ and use one of two different functions, either a cone shape,

$$
G_{\text {cone }}(\phi)=\mathbb{1}_{|\phi|<\phi_{0}}, 0 \leq \phi<2 \pi
$$

or a sinc shape $\mathrm{i}^{\mathrm{i}}$

$$
G_{\text {sinc }}(\phi)=\operatorname{sinc}^{2}\left(\frac{\phi}{\phi_{0}}\right), 0 \leq \phi<2 \pi .
$$

These are only crude approximations of true antenna directivity functions but suffice for the following derivations. The cone-shaped antenna gain function has the additional advantage of making the derivations in the following sections entirely analytical.

It must be emphasized that the choice of parameters is only relevant for the simulations; none of the derivations made in this paper are specific to a certain set of parameters, as long as the medium access occurs as described in Section 3.1 and the interferer positions can be modeled as a PPP. The signal parameter most relevant for this is the sub-carrier spacing, as it affects the Doppler tolerance of the OFDM signal (and thus guarantees orthogonality as postulated in Section 3.1). 


\section{Analytical results}

To analytically calculate the outage probability, we first reiterate on the assumptions and approximations made.

\subsection{Assumptions}

- The total, additive interference $\mathbf{Z}$ is modeled by WGN.

This implies a clock synchronization as discussed in Section 3.1, and that the data sent by the individual interferers are uncorrelated. Note that this is accurate for an OFDM radar system as described above; it merely requires the individual elements of $\mathbf{Z}$ to be i.i.d. Gaussian distributed (cf. Section 3.3) ${ }^{j}$.

- The medium access can be described by a transmit probability $p_{T x}$, and a transmitting node will randomly choose one of $U$ logical channels, each with equal probability (cf. Section 3.1).

- The attenuation between interfering transmitters and the reference system is modeled by path loss and a (random) fading coefficient, see Eq. (18).

These two assumptions might be less accurate for some specific scenario but are the most sensible assumptions if the OFDM radar system is not specified any closer.

- Nodes are distributed uniformly and independently.

This allows us to use the tools described in Section 4.3. It is motivated by the fact that due to the mobility of the nodes, their relative position changes all the time and is thus different between timeslots.

\subsection{Approximations}

- Sub-carriers are always orthogonal, interference on a different channel thus does not affect the radar system.

- An ideal radar system can always detect the reference target if $P_{\mathrm{Rx}} \cdot N M+\mathbb{E}\left[|z|^{2}\right]>\theta$, i.e., Eq. (13) is an equality.

These are the only simplifications in this model which are designed to facilitate the derivation of analytical bounds. Their influence is discussed in Section 5.2.

\subsection{Stochastic model}

Given the synchronized slotted medium access of the nodes, we consider the network in an arbitrarily chosen slot (snapshot). In this snapshot, the locations of the potentially interfering nodes are given by the PPP with density $\lambda$, as already explained in Section 3.3. From Slyvniak's theorem $[6,29]$, it follows that the law of the PPP is not changed by adding a node. Due to the stationarity, this node can be placed in the origin without loss of generality. We will refer to this node as the reference node as it will allow us to measure the typical performance in such a network.

Since the medium access is uncoordinated among the nodes, i.e., each node accesses the medium independently of each other with probability $p_{\mathrm{Tx}}$, we can obtain the set of interfering nodes by independent thinning of the original PPP [8]. The resulting point process is again Poisson with density $p_{\text {Tx }} \lambda$.

We treat the sub-carriers chosen by the $i$-th node as mark attached to this node. Formally, if node $i$ chooses the $\mathrm{u}$-th sub-carrier for transmission, we assign the mark $\mathrm{u}_{i}$ to this node. The small-scale channel fading experienced between the $i$-th interferer and our reference node is denoted by another mark, $g_{i}$.

Having introduced all relevant system parameters, we can now formally define the set of interferers by the stationary independently marked PPP

$$
\Phi:=\left\{\left(\mathrm{x}_{i}, \mathrm{u}_{i}, \mathrm{~g}_{i}\right)\right\}_{i=1}^{\infty}
$$

of (spatial) density $p_{\mathrm{Tx}} \lambda$, where the $\mathrm{x}_{i}$ denote the random interferer locations, and $\mathrm{u}_{i}$ and $\mathrm{g}_{i}$ are the marks associated with interferer $i$. Note that $\left(\mathrm{x}_{i}, \mathrm{u}_{i}, \mathrm{~g}_{i}\right) \in \mathbb{R}^{2} \times \mathbb{U} \times \mathbb{R}_{+}$, where $\mathbb{U}=\{1, \ldots, U\}$.

Without loss of generality, we assume that the reference node receives on the first channel, $u=1$. Then, the sum interference power measured at the reference node (in the origin) is given by

$$
\mathrm{Y}=\sum_{\left(\mathrm{x}_{i}, \mathrm{u}_{i}, \mathrm{~g}_{i}\right) \in \Phi} \mathbb{1}_{\left(\mathrm{u}_{i}=1\right)} \cdot G\left(\angle \mathrm{x}_{i}\right) \mathrm{g}_{i}\left\|\mathrm{x}_{i}\right\|^{-\alpha},
$$

where $\left\|\mathrm{x}_{i}\right\|^{-\alpha}$ describes the large-scale path loss between interferer location $\mathrm{x}_{i} \in \mathbb{R}^{2}$ and the origin, and $\angle \mathrm{x}_{i}$ the interferer's azimuth.

At the receiver, the sum interference is superimposed by thermal noise of power $\sigma_{N}^{2}$. As shown previously, the interference noise can be assumed to be conditionally AWGN. Consequently, the total noise is conditionally AWGN as well with a (random) power equal to $\tilde{Y}+\sigma_{N}^{2}$.

We must point out that $Y$ is a normalized, unit-less interference power term, which is introduced for its mathematical utility. On the other hand, $\tilde{Y}$ includes the physical effects, such as frequency-dependence of the free space path loss. Converting one value into another is done by

$$
\tilde{\mathrm{Y}}=\mathrm{Y} \cdot U \beta \text {. }
$$

The factor $\beta$ plays the same role as in Eq. (17). $U$ is necessary because the power on the individual sub-carriers is scaled by the same factor to retain a constant transmit power.

\subsection{Outage probability analysis}

From Section 3.2, we have that

$$
p_{\text {out }}=\operatorname{Pr}\left[P_{\text {peak }}<\theta\right] \text {. }
$$


In order to make use of stochastic geometry, we must express this probability in terms of Eq. (23), which we achieve by inserting Eqs. (9), (13), and (24). This results in

$$
\begin{aligned}
p_{\text {out }} & =\operatorname{Pr}\left[P_{\mathrm{Rx}} N M+U \beta \mathrm{Y}+\sigma_{N}^{2}<\left(U \beta \mathrm{Y}+\sigma_{N}^{2}\right) c\right] \\
& =\operatorname{Pr}[\mathrm{Y} \geq \underbrace{\frac{1}{U \beta}\left((c-1)^{-1} P_{\mathrm{Rx}} N M-\sigma_{N}^{2}\right)}_{\omega}] .
\end{aligned}
$$

Hence, computing $p_{\text {out }}$ requires the evaluation of the tail probability of $Y$ (note that Eq. (27) assumes that $c-1$ is positive, but unless $p_{F}$ is close to 1 , this is always the case). Unfortunately, solving Eq. (27) directly is an intractable problem; therefore, we present bounds in the following:

\subsubsection{Lower bound}

To obtain a lower bound, we make use of the dominant interferer phenomenon which was originally introduced for communication networks (see [7]), and adapt it to the case of radar network outage. The idea is to divide the set of total interferers into the set of dominant and nondominant interferers. Formally, these sets are defined as

$$
\Phi_{\mathrm{d}}:=\left\{\left(\mathrm{x}_{i}, \mathrm{u}_{i}, \mathrm{~g}_{i}\right) \in \Phi \mid \mathbb{1}_{\left(\mathrm{u}_{i}=1\right)} G\left(\angle \mathrm{x}_{i}\right) \mathrm{g}_{i}\left\|\mathrm{x}_{i}\right\|^{-\alpha} \geq \omega\right\}
$$

and

$$
\Phi_{\text {nd }}:=\left\{\left(\mathrm{x}_{i}, \mathrm{u}_{i}, \mathrm{~g}_{i}\right) \in \Phi \mid \mathbb{1}_{\left(\mathrm{u}_{i}=1\right)} G\left(\angle \mathrm{x}_{i}\right) \mathrm{g}_{i}\left\|\mathrm{x}_{i}\right\|^{-\alpha}<\omega\right\} .
$$

Note that $\Phi_{d} \cap \Phi_{\text {nd }}=\emptyset$. Accordingly, we define

$$
\mathrm{Y}_{\mathrm{d}}:=\sum_{\left(\mathrm{x}_{i}, \mathrm{u}_{i}, \mathrm{~g}_{i}\right) \in \Phi_{\mathrm{d}}} \mathbb{1}_{\left(\mathrm{u}_{i}=1\right)} G\left(\angle \mathrm{x}_{i}\right) \mathrm{g}_{i}\left\|\mathrm{x}_{i}\right\|^{-\alpha}
$$

and

$$
\mathrm{Y}_{\mathrm{nd}}:=\sum_{\left(\mathrm{x}_{i}, \mathrm{u}_{i}, \mathrm{~g}_{i}\right) \in \Phi_{\mathrm{nd}}} \mathbb{1}_{\left(\mathrm{u}_{i}=1\right)} G\left(\angle \mathrm{x}_{i}\right) \mathrm{g}_{i}\left\|\mathrm{x}_{i}\right\|^{-\alpha},
$$

where $Y=Y_{d}+Y_{\text {nd }}$. The intuition behind Eqs. (28) and (29) is that $\Phi_{d}$ contains those interferers that directly create outage individually, while $\Phi_{\text {nd }}$ contains interferers not directly creating outage.

The outage probability is thus

$$
p_{\text {out }}=\operatorname{Pr}\left[\mathrm{Y}_{\mathrm{d}}+\mathrm{Y}_{\mathrm{nd}}>\omega\right] \text {. }
$$

We can construct a simple lower bound by neglecting the $Y_{\text {nd }}$ term:

$$
\begin{aligned}
p_{\text {out }} \geq p_{\text {out }, \mathrm{d}} & :=\operatorname{Pr}\left[\mathrm{Y}_{\mathrm{d}}>\omega\right] \\
& =\operatorname{Pr}\left[\Phi_{\mathrm{d}} \neq \emptyset\right],
\end{aligned}
$$

where the equality stems from the fact that the presence of one dominant interferer already suffices to make the event $\mathrm{Y}>\omega$ true. Since $\Phi_{\mathrm{d}}$ is still a PPP, we have to compute the void probability of the Poisson distributed random variable $\left|\Phi_{\mathrm{d}}\right|$, i.e.,

$$
p_{\text {out }, \mathrm{d}}=1-\exp (-\mu),
$$

where the mean $\mu$ can be calculated using [8] as

$$
\begin{aligned}
\mu & =p_{\mathrm{Tx}} \lambda \int_{\mathbb{R}^{2}} \mathbb{E}\left[\mathbb{1}_{(\mathrm{u}=1)} \mathbb{1}_{\left(G(\angle x) \mathrm{g}\|x\|^{-\alpha} \geq \omega\right)}\right] \mathrm{d} x \\
& =p_{\mathrm{Tx}} \lambda \int_{\mathbb{R}^{2}} \operatorname{Pr}[\mathrm{u}=1] \operatorname{Pr}\left[G(\angle x) \mathrm{g}\|x\|^{-\alpha} \geq \omega\right] \mathrm{d} x \\
& =\frac{p_{\mathrm{Tx}} \lambda}{U} \int_{\mathbb{R}^{2}} \operatorname{Pr}\left[\mathrm{g} \geq \frac{\omega\|x\|^{\alpha}}{G(\angle x)}\right] \mathrm{d} x .
\end{aligned}
$$

At this point, the bound only depends on the probability distribution of the fading. For the fading cases discussed in Section 3.3, we can give solutions for $\mu$ :

Pure path $\operatorname{loss}(\mathbf{g} \equiv \mathbf{1})$ In this case,

$$
\operatorname{Pr}\left[\mathrm{g} \geq \frac{\omega\|x\|^{\alpha}}{G(\angle x)}\right] \rightarrow \mathbb{1}_{\left(\|x\| \leq\left(\frac{G(\angle x)}{\omega}\right)^{1 / \alpha}\right)},
$$

and therefore,

$$
\begin{aligned}
\mu & =\frac{2 p_{\mathrm{Tx}} \lambda}{U} \int_{0}^{\pi} \int_{0}^{\left(\frac{G(\phi)}{\omega}\right)^{\frac{1}{\alpha}}} r \mathrm{~d} r \mathrm{~d} \phi \\
& =\frac{p_{\mathrm{Tx}} \lambda}{U} \int_{0}^{\pi}\left(\frac{G(\phi)}{\omega}\right)^{\frac{2}{\alpha}} \mathrm{d} \phi .
\end{aligned}
$$

Rayleigh fading (g $\sim \operatorname{Exp}(1))$ Here, the probability is given by

$$
\operatorname{Pr}\left[g \geq \frac{\omega\|x\|^{\alpha}}{G(\angle x)}\right]=\exp \left(-\frac{\omega\|x\|^{\alpha}}{G(\angle x)}\right),
$$

and therefore, we obtain $\mu$ by solving

$$
\begin{aligned}
\mu & =\frac{2 p_{\mathrm{Tx}} \lambda}{U} \int_{0}^{\pi} \int_{0}^{\infty} r \exp \left(-\frac{\omega r^{\alpha}}{G(\phi)}\right) \mathrm{d} r \mathrm{~d} \phi \\
& =\frac{2 p_{\mathrm{Tx}} \lambda}{\alpha U} \int_{0}^{\pi} \int_{0}^{\infty} t^{\frac{2}{\alpha}-1} \exp \left(-\frac{\omega t}{G(\phi)}\right) \mathrm{d} t \mathrm{~d} \phi \\
& =\frac{p_{\mathrm{Tx}} \lambda}{U} \Gamma\left(1+\frac{2}{\alpha}\right) \int_{0}^{\pi}\left(\frac{G(\phi)}{\omega}\right)^{\frac{2}{\alpha}} \mathrm{d} \phi,
\end{aligned}
$$

where $\Gamma(\cdot)$ is the gamma function. Hence, we obtain the lower bound:

$$
\begin{aligned}
p_{\text {out }} & \geq p_{\text {out }, \mathrm{d}} \\
& =1-\exp \left(-\frac{p_{\mathrm{Tx}} \lambda}{U} \omega^{-\frac{2}{\alpha}} \Gamma\left(1+\frac{2}{\alpha}\right) \int_{0}^{\pi} G(\phi)^{\frac{2}{\alpha}} \mathrm{d} \phi\right)
\end{aligned}
$$


The only difference between the Rayleigh fading and the path-loss-only scenario is the $\Gamma\left(1+\frac{2}{\alpha}\right)$ function, which is not present for no fading.

Approximating the directivity At this point, the lower bound still depends on an integral over $G(\phi)$. If $G(\phi)$ is known, the integral may be solved analytically. Otherwise (e.g., if the antenna gain is only given in tabular form), the integral must be solved numerically. We will show the former case for the cone-shaped antenna function $G_{\text {cone }}(\phi)$ and Rayleigh fading, where the lower bound becomes very simple:

$$
p_{\text {out }} \geq p_{\text {out,d }}=1-\exp \left(-\frac{p_{\mathrm{Tx}} \lambda \phi_{0}}{U} \omega^{-\frac{2}{\alpha}} \Gamma\left(1+\frac{2}{\alpha}\right)\right) .
$$

\subsubsection{Upper bound}

Because both the dominant and non-dominant interferers can cause outage, we can write

$$
\begin{aligned}
p_{\text {out }} & =\operatorname{Pr}\left[\mathrm{Y}_{\mathrm{d}} \geq \omega\right]+\operatorname{Pr}\left[\mathrm{Y}_{\mathrm{d}}<\omega\right] \operatorname{Pr}\left[\mathrm{Y}_{\text {nd }} \geq \omega\right] \\
& =p_{\text {out }, \mathrm{d}}+\left(1-p_{\text {out }, \mathrm{d}}\right) \operatorname{Pr}\left[\mathrm{Y}_{\text {nd }} \geq \omega\right]
\end{aligned}
$$

where the second equation stems from the fact that dominant interferers always cause outage when present.

We obtain an upper bound on $p_{\text {out }}$ by applying Markov's inequality [30],

$$
\operatorname{Pr}\left[\mathrm{Y}_{\mathrm{nd}} \geq \omega\right] \leq \frac{1}{\omega} \mathbb{E}\left[\mathrm{Y}_{\mathrm{nd}}\right]
$$

We therefore focus on the first moment of $Y_{\text {nd }}$ : Using Campbell's theorem [6], we can compute $\mathbb{E}\left[\mathrm{Y}_{\text {nd }}\right]$ as follows:

$$
\begin{aligned}
\mathbb{E}\left[\mathrm{Y}_{\mathrm{nd}}\right] & =\mathbb{E}\left[\sum_{\left(\mathrm{x}_{i}, \mathrm{u}_{i}, \mathrm{~g}_{i}\right) \in \Phi_{\mathrm{nd}}} \mathbb{1}_{\left(\mathrm{u}_{i}=1\right)} \mathbb{1}_{\left(G(\angle x) \mathrm{g}\|x\|^{-\alpha}<\omega\right)} G\left(\angle \mathrm{x}_{i}\right) \mathrm{g}_{i}\left\|\mathrm{x}_{i}\right\|^{-\alpha}\right] \\
& =p_{\mathrm{Tx}} \lambda \int_{\mathbb{R}^{2}} \mathbb{E}\left[\mathbb{1}_{(\mathrm{u}=1)} \mathbb{1}_{\left(\mathrm{g}<\frac{\omega\|x\| \alpha}{G(\angle x)}\right)} G(\angle x) \mathrm{g}\|x\|^{-\alpha}\right] \mathrm{d} x .
\end{aligned}
$$

This is further simplified by making use of the fact that the marks are independent and splitting up the mean,

$$
\begin{aligned}
\mathbb{E}\left[\mathrm{Y}_{\mathrm{nd}}\right]= & p_{\mathrm{Tx}} \lambda \int_{\mathbb{R}^{2}} \operatorname{Pr}[\mathrm{u}=1] \mathbb{E}\left[\mathbb{1}_{\left(\mathrm{g}<\frac{\omega\|x\|^{\alpha}}{G(\angle x)}\right)} \mathrm{g}\right] \\
& \times G(\angle x)\|x\|^{-\alpha} \mathrm{d} x \\
= & \frac{p_{\mathrm{Tx}} \lambda}{U} \int_{\mathbb{R}^{2}} G(\angle x)\|x\|^{-\alpha} \int_{0}^{\frac{\omega\|x\|^{\alpha}}{G(\angle x)}} g \mathrm{dPr}[\mathrm{g}<g] \mathrm{d} x .
\end{aligned}
$$

Pure path loss $(\mathbf{g} \equiv \mathbf{1})$ The inner integral becomes $\mathbb{1}_{\left(\|x\| \geq\left(\frac{G(\angle x)}{\omega}\right)^{\frac{1}{\alpha}}\right)}$ and thus

$$
\begin{aligned}
\mathbb{E}\left[\mathrm{Y}_{\mathrm{nd}}\right] & =\frac{2 p_{\mathrm{Tx}} \lambda}{U} \int_{0}^{\pi} G(\phi) \int_{0}^{\infty} r^{-\alpha+1} \mathbb{1}_{\left(r \geq\left(\frac{G(\phi)}{\omega}\right)^{\frac{1}{\alpha}}\right)} \mathrm{d} r \mathrm{~d} \phi \\
& =\frac{2 p_{\mathrm{Tx}} \lambda}{U} \int_{0}^{\pi} G(\phi) \int_{\left(\frac{G(\phi)}{\omega}\right)^{\frac{1}{\alpha}}}^{\infty} r^{-\alpha+1} \mathrm{~d} r \mathrm{~d} \phi \\
& =\frac{2 p_{\mathrm{Tx}} \lambda}{U(\alpha-2)} \int_{0}^{\pi} G(\phi)\left(\frac{G(\phi)}{\omega}\right)^{\frac{2}{\alpha}-1} \mathrm{~d} \phi
\end{aligned}
$$

In this case, the tail probability $\operatorname{Pr}\left[\mathrm{Y}_{\text {nd }} \geq \omega\right]$ is bounded as

$$
\operatorname{Pr}\left[\mathrm{Y}_{\mathrm{nd}} \geq \omega\right] \leq \frac{2 p_{\mathrm{Tx}} \lambda \omega^{-\frac{2}{\alpha}}}{U(\alpha-2)} \int_{0}^{\pi} G(\phi)^{\frac{2}{\alpha}} \mathrm{d} \phi .
$$

By inserting this into Eq. (48), we obtain the upper bound

$$
p_{\text {out }} \leq p_{\text {out }, \mathrm{d}}+\left(1-p_{\text {out }, \mathrm{d}}\right) \frac{2 p_{\mathrm{Tx}} \lambda \omega^{-\frac{2}{\alpha}}}{U(\alpha-2)} \int_{0}^{\pi} G(\phi)^{\frac{2}{\alpha}} \mathrm{d} \phi .
$$

\subsection{Empirical verification}

\subsubsection{Verification of bounds}

In order to verify the bounds, we use the following simulation:

1. For every density $\lambda$, a radius $R$ was chosen such that the average number of nodes satisfies $\frac{\lambda}{U} \pi R^{2} \geq 1,000$, but the radius is not less than $R \geq 200 \mathrm{~m}$. For all simulations, the value $\lambda$ is interpreted as average number of nodes per square meter.

2. A number $I$ of interferers was randomly chosen from a Poisson distribution with mean parameter $p_{\mathrm{Tx}} \lambda \pi R^{2}$. Each of these interferers was assigned a random position $x$ uniformly on the disc with radius $R$.

3. The total interference power for this realization is thus

$$
P_{\mathrm{Ix}}=\sum_{i=0}^{I-1} \mathrm{~g}_{i} \frac{\beta G\left(\angle x_{i}\right)}{\|x\|_{i}^{\alpha}},
$$

using $\beta$ from Eq. (19).

4. If $P_{\text {peak }}>P_{\mathrm{Ix}}+\sigma_{N}^{2}$ is true, this scenario counts as a detection. For every set of parameters, 10,000 realizations were run to estimate the outage probability as the fraction of scenarios where the target was not detected.

Figure 4 shows some simulation results, together with the upper and lower bounds. Note that both $p_{D}$ and $p_{\text {out }}$ are displayed, as the detection rate is the more intuitive metric. The lower bound for $p_{\text {out }}$ becomes an upper 


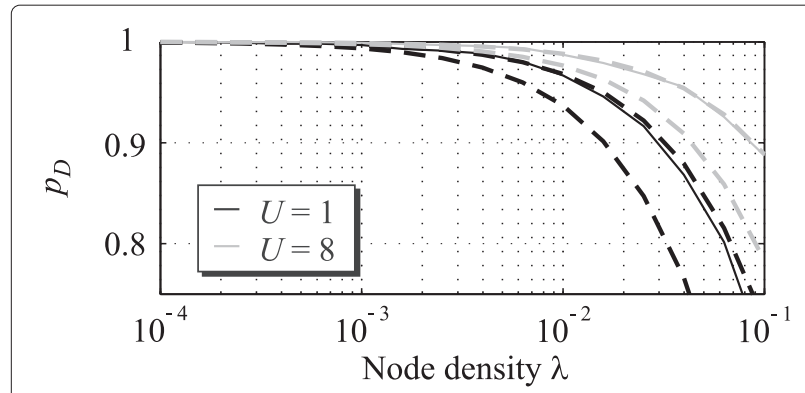

(a)

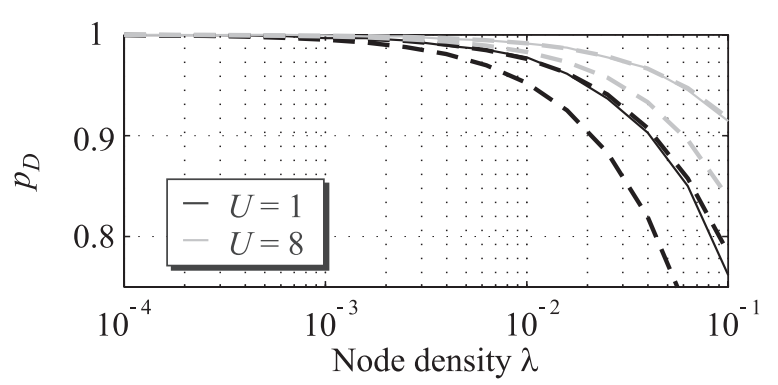

(b)

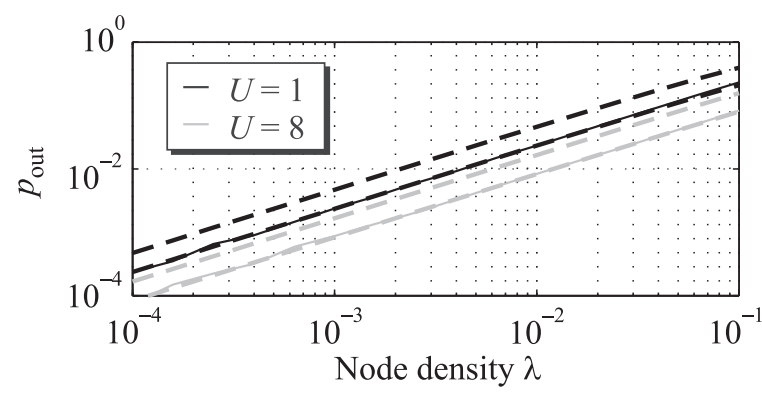

(c)

Figure 4 Simulated results (solid lines) and bounds (dashed lines) for $\alpha=4, \phi_{0}=\pi / 2$, and varying node densities. (a) Cone-shaped antenna function. (b) Sinc-shaped antenna function. (c) Sinc-shaped antenna function (outage probability).

bound for $p_{D}$, and vice versa. We cannot only see that the bounds are correct but also that the upper bound is very tight and can be used as a good approximation of the actual detection probability. The tightness of the upper bound results from the fact that, especially at high detection rates (which typically is the desired region of operation in practical car-safety applications), the 'dominant interferer' effect outweighs the sum interference created by the non-dominant interferers. This concurs with other research using stochastic geometry $[7,10]$.

Figure 5 shows the same simulation but with a variation of $\alpha$ instead of $\lambda$. When $\alpha$ approaches 2, the overall interference increases, as nodes from further away become more and more influential. We can observe that here,

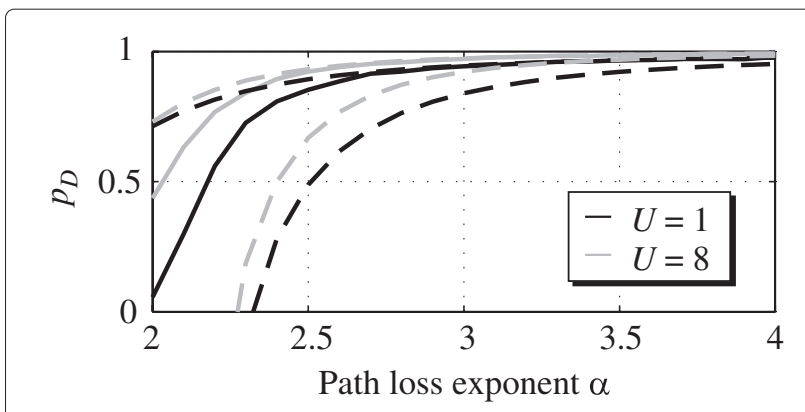

Figure 5 Simulated results (solid lines) and bounds (dashed lines) for $\lambda=10^{-2}, \phi_{0}=\pi / 2$, and sinc-shaped antenna gain.

too, the bound becomes less tight for higher interference levels.

\subsubsection{Model robustness for real estimates}

The previous simulation assumes perfect detection; i.e., the reference target is guaranteed to be detected when $P_{\text {peak }}>\theta$. More realistic simulations are required to verify if the model works with the signal processing presented in Section 2 and the approximations shown in Section 4.1.

However, it is important to separate the detection from the multi-target identification, which will be discussed in Section 5.2. A target identification system has to distinguish sidelobes from targets, handle the case where targets are very close and have overlapping main lobes, etc., and therefore is an additional source of errors. To simulate an OFDM radar system without introducing multi-target-related errors, we create a scenario where everything except the reference target has zero RCS, and the reference target is therefore the only scatterer. The interferers only produce interference noise.

The simulation works as follows: For every iteration, a number of interferers (and their positions) is modeled the same way as in Section 4.5.1. The reference node, as well as every active interferer node, transmit an OFDM frame. The signal of the reference node is attenuated according to Eq. (14) and delayed by $\tau=2 \frac{r_{\text {Ref }}}{c_{0}}$. The interference signals are attenuated according to their azimuth and antenna function as well as the path loss (with $\alpha=4$ ). To reduce the amount of random effects, no small-scale fading is applied here.

All signals (reflected reference signal and all interferer signals) and the thermal noise are added up and passed to an estimator that works as described in Section 2.2. We confirm a detection by checking that the periodogram bin corresponding to the reference target's distance and Doppler $n_{0}=\frac{r_{\text {Ref }}}{c_{0}} 2 \Delta f N_{\text {Per }}$ (rounded to the nearest integer) is larger than the threshold, $\operatorname{Per}\left(n_{0}, 0\right)>\theta$. At every simulation point, 1,000 iterations were run. 


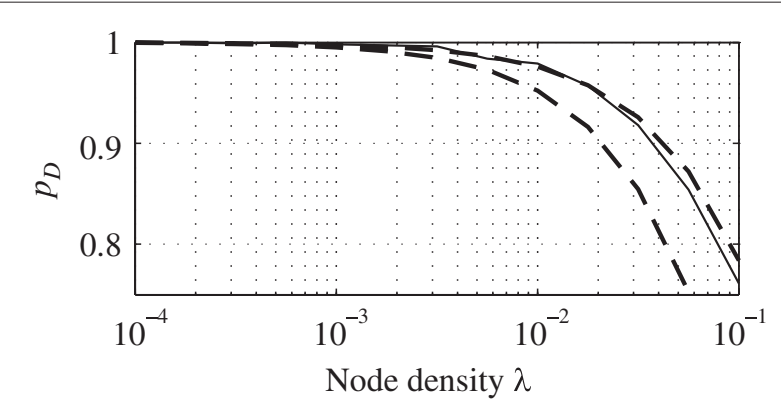

Figure 6 Simulation of the ideal detector $(\alpha=4$, cone-shaped antenna function, $\phi_{0}=\pi / 2, U=1$, no fading). The solid line shows the simulation results, the dashed lines the analytical upper and lower bounds.

Figure 6 shows the simulation results. Note that the simulated curve again stays very close to the upper bound. This confirms that the approximation (13) is justified, as the simulated curves from Figures 6 and 4 are very close to each other.

Because this simulation omits all multi-target-related errors, it is called an ideal detector in the following. It is clear that such a detector cannot be implemented in reality, where there are many targets with non-zero RCS.

\section{Consequences for the OFDM radar network parametrization}

As an approximation for the outage probability, the bounds can be an extremely useful tool. The following section gives some practical examples of their utility.

\subsection{Network feasibility study}

The most obvious use for these new metrics is a feasibility check of a given radar network to determine whether or not a network would fulfill certain QoS requirements. In this case, we can take a set of system parameters and use the bounds for the detection probability to see if the radar system is expected to work reliably.

As an example, consider the results from Figure 4. Say that we require a detection probability of $99 \%$ for safety reasons, the node density cannot increase beyond $\lambda=$ $10^{2.2}$, which corresponds to one node per $158.5 \mathrm{~m}^{2}$ on average. In a vehicular scenario, this corresponds to an average of one vehicle equipped with an OFDM radar system every $53 \mathrm{~m}$ for a lane width of $3 \mathrm{~m}$, which seems realistic given that most likely, not all vehicles would be equipped with such a radar system. For other node densities, the corresponding detection probability can be read from Figure 4. If the radar system must work with a specific detection probability with a higher node density, the parametrization can be changed in several ways:

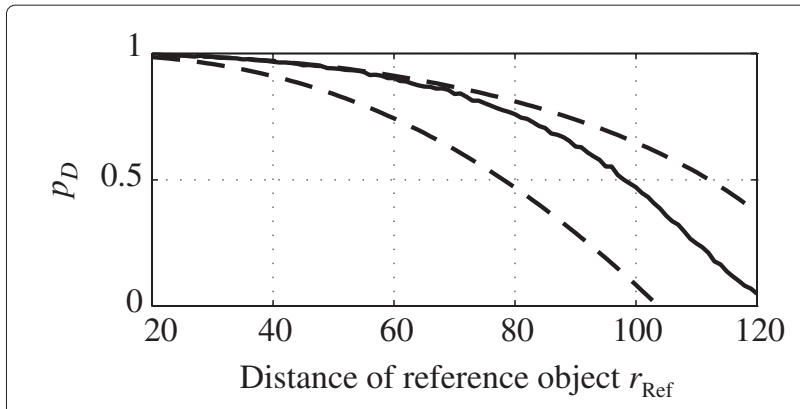

Figure 7 Detection probability over distance of the reference object. $\alpha=3, \lambda=10^{-2}$, sinc-shaped antenna gain with $\phi_{0}=\pi / 2$.

Either the number of channels $U$ is increased, or $\phi_{0}$ is reduced.

This also works the other way: given a node density $\lambda$ and a minimum detection probability, what is the smallest target the radar can detect? We can calculate the detection probability for different values of $P_{\text {peak }}$ to answer this question. Figure 7 shows $p_{D}$ as a function of $r_{\text {Ref }}\left(P_{\text {peak }}\right.$ is recalculated for every value of $r_{\text {Ref }}$ using Eq. (13), Eq. (14), and the values from Section 3.5). This time, the $P_{\mathrm{Rx}, \mathrm{Ref}}$ is reduced instead of increasing the average interference power. We see this has a similar effect concerning the bounds.

It is worth pointing out the analogy to the outage capacity of communication networks, which is a data rate that can be achieved with a given probability. Here, a target with peak amplitude $P_{\text {peak }}$ can be detected with probability $p_{D}$.

So far, this could be achieved by simulations - although time-consuming, the results would be very similar. To show the benefits of using the lower bound for $p_{\text {out }}$ as an approximation, we take our previous result (56)

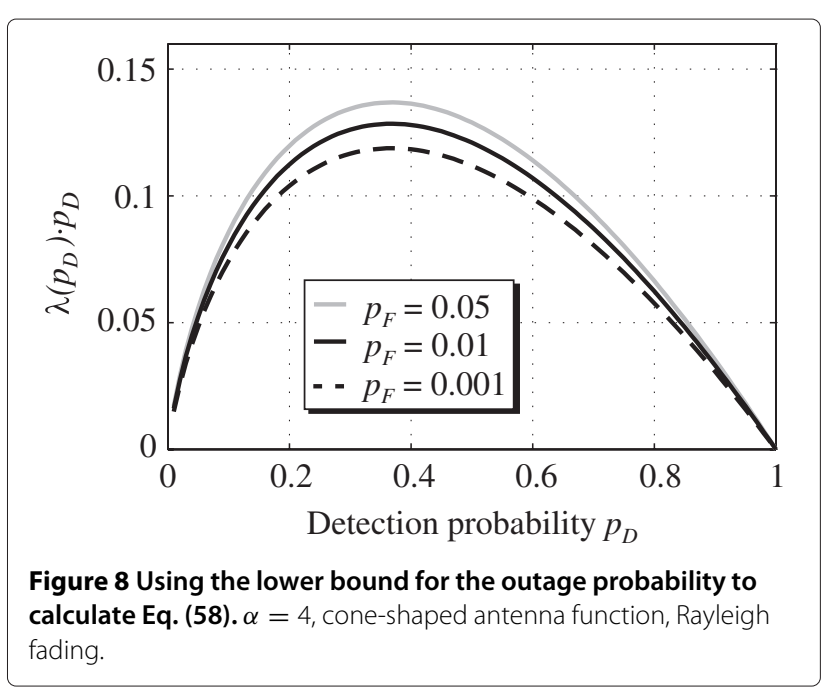


(cone-shaped antenna function, Rayleigh fading) and solve it for $\lambda$ :

$$
\lambda\left(p_{D}\right)=\frac{-\log \left(p_{D}\right) U}{p_{\mathrm{Tx}} \phi_{0} \omega^{-\frac{2}{\alpha}} \Gamma\left(1+\frac{2}{\alpha}\right)} .
$$

This allows us to first fix a tolerable detection probability and then obtain a maximum node density from that. Using (58), we can now plot the expected density of successfully detecting nodes $\left(p_{D} \cdot \lambda\left(p_{D}\right)\right)$ as a function of the required detection probability. Figure 8 shows this value for different values of $p_{F}$.

This demonstrates how these simple bounds provide a powerful tool for benchmarking the network performance of an OFDM radar system.

\subsection{Evaluation of the detection performance}

Section 4.5 discussed how well an ideal detector would perform. This can be used as a benchmark for practical implementations.

To demonstrate this, the simulation in Section 4.5.2 was modified to a more realistic scenario: First, every interferer is assigned a random RCS which obeys an exponential distribution with pdf $p\left(\sigma_{\mathrm{RCS}}\right)=\frac{1}{\bar{\sigma}_{\mathrm{RCS}}} e^{\frac{-\sigma_{\mathrm{RCS}}}{\bar{\sigma}_{\mathrm{RCS}}}}, \sigma_{\mathrm{RCS}}>0$. The mean RCS was chosen as $\bar{\sigma}_{\mathrm{RCS}}=10 \mathrm{~m}^{2}$.

Next, the radar signal processing uses a modified CLEAN algorithm [31] to estimate Doppler and range of all interferences and the reference target. This algorithm detects the largest peak in the periodogram, estimates range and Doppler, and then subtracts the components from the periodogram caused by the estimated values. This process is repeated until the largest remaining peak is smaller than $\theta$. To make sure peaks are not caused by residuals during the subtraction process, peaks detected within the main lobe of a previously detected target are discarded during the process.

For large $\lambda$, the probability of a large target being in close proximity to the reference target increases, in which case the reference target might be overshadowed and therefore not detected.

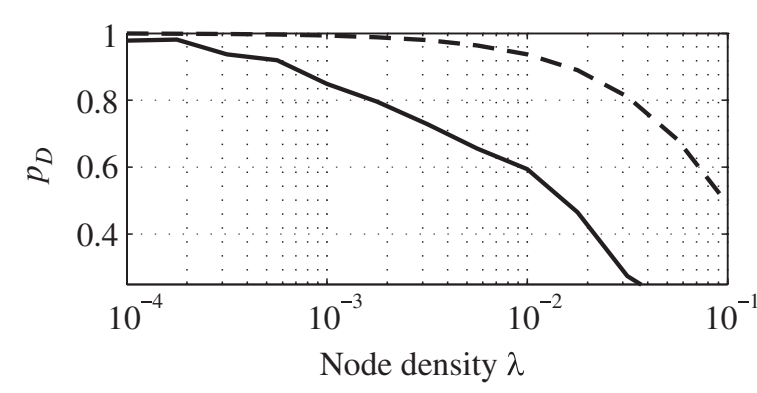

Figure 9 Simulation using the cancelation algorithm ( $\alpha=4$, cone-shaped antenna, $\phi_{0}=\pi / 2, U=1$, no fading). Only the upper bound is displayed (dashed line).
The simulation results in Figure 9 confirm this (note that we only consider detection of the reference target; the detection probability of the other nodes is not a useful metric as their positions are random). We can identify a significant reduction of the detection probability compared to the ideal detector, although it must be noted that for higher node densities, the detector is forced to handle several hundred clutter objects (the interferers), which is a difficult task for radar systems in general. The root mean square (RMS) error of the estimation was also measured during the simulations, but as a successful detection is a prerequisite of calculating an error, the RMS error constantly stays below $0.6 \mathrm{~m}$ for the range and below $0.6 \mathrm{~m} / \mathrm{s}$ for the Doppler estimation.

Using Eq. (56), we can quantify the performance reduction of the successive cancelation algorithm compared to the ideal detector for a certain configuration. From Figure 9, we inspect the situation for $\lambda=10^{-3}$ and find that at this density, we achieve a detection probability of $p_{D}=0.85$. To quantify the detector loss, we solve Eq. (46) for $P_{\mathrm{Rx}, \text { ref }}$ and determine which reflected energy would cause the ideal detector to have the same detection probability (we use the upper bound as the value for the ideal detector):

$$
P_{\mathrm{Rx}, \text { detector }}=\frac{c-1}{N M}\left(\left(\frac{P_{\mathrm{Tx}} \lambda \phi_{0}}{U \log \left(1-p_{D}\right)}\right)^{\frac{\alpha}{2}} U \beta+\sigma_{N}^{2}\right)
$$

By comparing the received power with the reflected power of the reference object, we may define the detector loss as

$$
L=\frac{P_{\mathrm{Rx}, \text { detector }}}{P_{\mathrm{Rx}, \mathrm{ref}}} .
$$

For the values used to create Figure 9, we obtain a received power of $-127 \mathrm{dBm}$, or an $18-\mathrm{dB}$ detector loss. Other multi-target detection algorithms might achieve better values, but we now have a practical limit for how good such an algorithm can get.

We emphasize that this benchmark is only valid for a fixed scenario.

\subsection{Choice of sub-carrier spacing}

In Section 3.1, we introduce the possibility of using a carrier spacing method $(U>1)$ to allow multiple user access to the medium. This has two effects: first, it decreases the chance of a collision, as different users may access the medium on different logical channels. On the other hand, whenever a collision occurs, the influence of the interfering signal is in fact worse than it were for $U=1$, because the signal power per active carrier is increased by a factor $U$. This requires a closer analysis on how the choice of $U$ affects the multi-user performance. 
These results provide a simple answer for this: consider Eq. (46), where $U$ appears twice: once in the PPP density $\left(p_{\mathrm{Tx}} \lambda / U\right)$, and once in $\omega^{\frac{2}{\alpha}}$. The argument of the exponential function therefore depends on $U^{-\left(\frac{2}{\alpha}-1\right)}$. Because $\frac{2}{\alpha}-1>0$, the outage probability will always decrease for higher $U$, and this can thus be chosen as large as external constraints permit.

\section{Conclusions}

In this paper, we have suggested a novel figure of merit for radar systems: the radar outage probability. This is a scalar value which describes the capability to detect a specific target. It depends on the density of nodes and the waveform parameters.

More importantly, we could derive an asymptotically tight bound, which provides an analytical expression of radar systems without simulations or measurement campaigns (although empirical methods are used to verify the results). As an example, we can calculate that the OFDM radar system suggested in [3] would have an outage probability of less than $1 \%$ for traffic densities below one node per $158.5 \mathrm{~m}^{2}$.

Despite being a theoretical model, only few assumptions and approximations are made (see the introduction of Section 4). This makes the result applicable in practice, e.g., as a quick, computationally inexpensive tests to verify if a given waveform achieves a certain outage probability.

It can also be used to benchmark implementations of radar systems. The complementary probability of $p_{\text {out }}=$ $1-p_{D}$ is an ideal detection rate. If the measured detection rate lies below that, the loss due to the implementation can be quantified.

\section{Endnotes}

a Multiple radar sensors working cooperatively are sometimes also referred to as radar networks. Here, the network aspect describes the distributed nature of nodes which can also communicate among each others.

b We assume that the reader is familiar with OFDM; for a more detailed introduction, we refer to standard textbooks, e.g., [32,33]. OFDM radar, in particular the signal processing components, is explained in [3].

c Here, anything backscattering energy is considered, be it a regular target or clutter.

${ }^{d}$ In [3], the authors discuss the usage of a Hamming window.

e This is further discussed in Section 5.2.

${ }^{\mathrm{f}}$ In the context of vehicular technology, we can safely assume the existence of a GPS receiver.

$\mathrm{g}$ In such a point process, points (here, nodes) are independently distributed at random in a given area; on average, there are $\lambda$ nodes per unit area. For an introduction to PPPs, cf. [6-8]. $\mathrm{h}$ The fading of the nodes is identically distributed, the distribution itself does not depend on $i$.

${ }^{\mathrm{i}} \operatorname{sinc}(x)=\sin (\pi x) / \pi x$

j This does not state that the individual analog transmit signals are pure white Gaussian noise, rather that the superposition of such signals in the discrete-time domain are uncorrelated.

\section{Competing interests}

The authors declare that they have no competing interests.

Received: 25 March 2013 Accepted: 22 July 2013

Published: 14 August 2013

\section{References}

1. C Sturm, T Z wick, W Wiesbeck: An OFDM system concept for joint radar and communications operations, in Vehicular Technology Conference, 2009. 69th IEEE. Barcelona, 26-29 April 2009

2. M Takeda, TTerada, R Kohno, Spread spectrum joint communication and ranging system using interference cancellation between a roadside and a vehicle, in Vehicular Technol Conf., 1998. VTC 98. 48th IEEE 3, Ottawa, 18-21 May 1998, pp.1935-1939

3. C Sturm, W Wiesbeck, Waveform design and signal processing aspects for fusion of wireless communications and radar sensing. Proc. IEEE. 99(7), 1236-1259 (2011)

4. More Safetey for All by Radar Interference Mitigation (MOSARIM). 7th Frame EU-Project, FP7/2007-2013. http://www.mosarim.eu. Accessed 30 July 2013

5. M Braun, C Sturm, FK Jondral, On the Single-Target Accuracy of OFDM Radar Algorithms, in 22nd IEEE Symposium on Personal Indoor and Mobile Radio Communications, Toronto, 1-14 September 2011

6. D Stoyan, W Kendall, J Mecke, Stochastic Geometry and its Applications, 2 edition (Wiley, Hoboken, 1995)

7. S Weber, X Yang, J Andrews, G de Veciana, Transmission capacity of wireless ad hoc networks with outage constraints. IEEE Trans. Inf. Theory. $\mathbf{5 1}, 4091-4102$ (2005)

8. F Baccelli, B Blaszczyszyn, Stochastic geometry and wireless networks, volume 1+2: theory and applications. Foundations and TrendS ${ }^{\oplus i n}$ Networking. 4, 1-312 (2009)

9. R Tanbourgi, H Jäkel, L Chaichenets, F Jondral, Interference and throughput in Aloha-based ad hoc networks with isotropic node distribution, in IEEE Int. Symposium Inf. Theory (ISIT), Cambridge, MA, 1-6 July 2012, pp. 616-620

10. R Tanbourgi, $\mathrm{H}$ Jäkel, F Jondral, Interference and Throughput in Poisson Networks with Isotropically distributed Nodes. Submitted, available at: http://arxiv.org/abs/1211.4755. Accessed 30 July 2013

11. G Brooker, Mutual interference of millimeter-wave radar systems. Electromagnetic Compatibility, IEEE Trans. 49, 170-181 (2007)

12. M Goppelt, HL Blocher, W Menzel, Analytical investigation of mutual interference between automotive FMCW radar sensors, in Microwave Conference (GeMIC), 2011 German, Darmstadt, 14-16 March 2011, pp.1-4

13. D Oprisan, $\mathrm{H}$ Rohling, Analysis of mutual interference between automotive radar systems, in Proc. International Radar Symposium (2005), Bangalore, 19-22 December 2005, p.84

14. TM Consortium, Study on the state-of-the-art interference mitigation techniques. Work Package 1, General Interference Risk Assessment (European Commission, Bruxelles, 2010)

15. Y Sit, L Reichardt, C Sturm, T Zwick, Extension of the OFDM joint radar-communication system for a multipath, multiuser scenario, in Radar Conference (RADAR), 2011 IEEE (2011), Kansas City, 23-27 May 2011, pp. 718-723

16. M Hischke, Collision warning radar interference, in Proceedings of the Intelligent Vehicles '95 Symposium, Detroit, 25-26 September 1995 pp. 13-18

17. T Schipper, M Harter, L Z wirello, T Mahler, T Zwick, Systematic approach to investigate and counteract interference-effects in automotive radars, in Radar Conference (EURAD), 2012 9th European, Amsterdam, 31 October-02 November 2012, pp. 190-193 
18. L Reichardt, J Maurer, T Fügen, T Zwick, Virtual Drive: a Complete V2X Communication and Radar System Simulator for Optimization of Multiple Antenna Systems. Proc. IEEE. 99(7), 1295-1310 (2011)

19. J Karedal, F Tufvesson, N Czink, A Paier, C Dumard, T Zemen, C Mecklenbrauker, A Molisch, A geometry-based stochastic MIMO model for vehicle-to-vehicle communications. Wireless Commun. IEEE Trans. 8(7), 3646-3657 (2009)

20. M Braun, C Sturm, FK Jondral, Maximum likelihood speed and distance estimation for OFDM radar. Radar Conf., IEEE Int., Arlington. 10-14 May 2010

21. SM Kay, Modern Spectral Estimation (Prentice Hall, Upper Saddle River, 1988)

22. MIE Skolnik (ed.), Radar Handbook, 2. ed. edition (Knovel, Norwich, 2003)

23. C Sturm, YL Sit, M Braun, T Zwick, Spectrally interleaved multi-carrier signals for radar network applications and MIMO-radar. IET Radar, Sonar Navigation. 7, 261-269 (2013)

24. A Goldsmith, Wireless Communications (Cambridge University Press, Cambridge, 2005)

25. J Andrews, R Ganti, M Haenggi, N Jindal, S Weber, A primer on spatial modeling and analysis in wireless networks. IEEE Commun. Mag. 48(11), 156-163 (2010)

26. W Hengartner, R Theodorescu, Einführung in die Monte-Carlo-Methode (Carl Hanser Verlag, Munich, 1978)

27. C Sturm, M Braun, T Zwick, W Wiesbeck, Performance Verification of Symbol-Based OFDM Radar Processing, in IEEE Int. Radar Conf., Washington, DC, 10-14 May 2010

28. M Braun, C Sturm, A Niethammer, FK Jondral, Parametrization of joint OFDM-based radar and communication systems for vehicular applications, in 20th IEEE Symposium on Personal Indoor and Mobile Radio Communications, Tokyo, 13-16 September 2009

29. A Baddeley, Spatial point processes and their applications, in Stochastic Geometry: Lectures given at the C.I.M.E. 2004, Lecture Notes in Mathematics 1892 (Springer Verlag, Heidelberg, 2006), pp. 1-75

30. A Gut, Probability: A Graduate Course (Springer, New York, 2005)

31. J Tsao, B Steinberg, Reduction of sidelobe and speckle artifacts in microwave imaging: the CLEAN technique. Antennas Propagation, IEEE Trans. 36(4), 543-556 (1988)

32. L Hanzo (ed.), OFDM and MC-CDMA for Broadband Multi-user Communications, WLANs and Broadcasting (Wiley, Hoboken, 2003)

33. M Engels (ed.), Wireless OFDM Systems: How to Make Them Work? (Kluwer Academic Publ, Dordrecht, 2002)

doi:10.1186/1687-1499-2013-207

Cite this article as: Braun et al:: Co-channel interference limitations of OFDM communication-radar networks. EURASIP Journal on Wireless Communications and Networking 2013 2013:207.

\section{Submit your manuscript to a SpringerOpen ${ }^{\circ}$ journal and benefit from:}

- Convenient online submission

- Rigorous peer review

- Immediate publication on acceptance

- Open access: articles freely available online

- High visibility within the field

- Retaining the copyright to your article

Submit your next manuscript at $\boldsymbol{\wedge}$ springeropen.com 